\section{Commentary: Cardiac surgery during the coronavirus disease 2019 (COVID-19) pandemic: Feeling our way in the dark}

\author{
Clifford W. Barlow, FRCS (CTh), DPhil
}

The coronavirus disease 2019 (COVID-19) pandemic has presented unprecedented challenges to international health care systems. While cardiac surgeons have been deployed to assist in treating nonsurgical patients with COVID-19, a major challenge has been in providing cardiac surgical treatment that keeps both patients and staff safe from COVID-19 infection. However, with no recommendations available to guide current decisionmaking, there is significant variation in practice, and only time will reveal the impact of the pandemic on patient outcomes.

In this issue of the Journal, Benedetto and colleagues ${ }^{1}$ appraised UK consultant surgeons' opinions on clinical decision-making to generate provisional recommendations for cardiac surgery during the COVID-19 pandemic. A Web-based questionnaire was sent to all consultant cardiac surgeons, and responses were received from 86 of 198 surgeons. The authors defined a "strong consensus" as an opinion shared by at least $60 \%$ of responders and also considered whether surgeons worked in units in which resources were entirely, partially, or not at all redirected to treat patients with COVID-19.

Responses were varied, revealing considerable uncertainty even within a single country and health care system. Nevertheless, there was strong agreement that the risk of COVID-19 exposure for patients undergoing heart surgery

\footnotetext{
From the Department of Cardiothoracic Surgery, University Hospital Southampton, Southampton, United Kingdom.

Disclosures: The author reported no conflicts of interest.

The Journal policy requires editors and reviewers to disclose conflicts of interest and to decline handling or reviewing manuscripts for which they may have a conflict of interest. The editors and reviewers of this article have no conflicts of interest.

Received for publication May 26, 2020; revisions received May 26, 2020; accepted for publication May 27, 2020; available ahead of print June 6, 2020.

Address for reprints: Clifford W. Barlow, FRCS (CTh), DPhil, Department of Cardiothoracic Surgery, University Hospital Southampton, Tremona Rd, Southampton, United Kingdom SO16 6YD (E-mail: cliffbarlow@hotmail.com).

J Thorac Cardiovasc Surg 2020;160:976-7 0022-5223/ $\$ 36.00$

Crown Copyright (C) 2020 Published by Elsevier Inc. on behalf of The American Association for Thoracic Surgery

https://doi.org/10.1016/j.jtcvs.2020.05.086
}

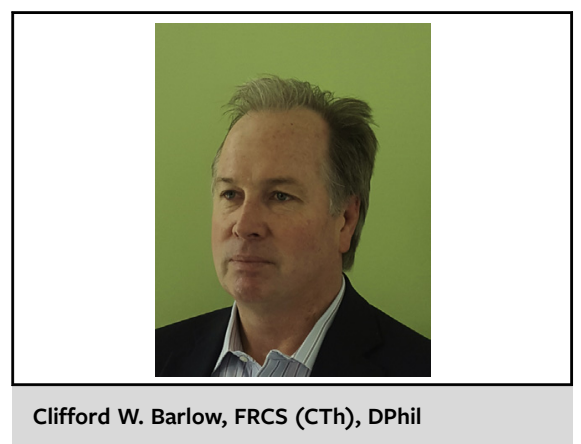

CENTRAL MESSAGE

The views of senior surgeons can provide recommendations for clinical decision-making for cardiac surgery during the COVID-19 pandemic while clinical data are awaited.

could be moderate to high and likely to increase mortality if it occurs. As a result, personal protective equipment should be adopted in every case by the operating room team regardless of the patient's COVID-19 status, and all cardiac procedures should be decided based on an ad-hoc multidisciplinary team. The responders strongly supported that, before hospital admission, every patient should receive a nasopharyngeal swab, polymerase chain reaction and, more surprisingly, computed tomography (CT) of the chest. The majority $(>50 \%)$ agreed that patients who tested positive for COVID-19 before salvage surgery (ie, dissection) should be considered for surgery only if they have no symptoms of infection and that aortic and mitral valve surgery should similarly be considered only in selected cases. Opinion about who should have coronary artery bypass graft surgery was much more varied. Most pleasingly, there was strong agreement that this pandemic will not have a long-term impact on surgical activities.

The study by Benedetto and colleagues ${ }^{1}$ provides important and interesting information. Although the authors describe some recommendations as a "consensus," which implies a degree of discussion and agreement, and many of them echo existing national and international recommendations, the views of senior surgeons can represent an instrument to guide heath policy makers. UK units were unequally represented, some with only one responder and others with several who possibly all reflected the same locally agreed protocols. Nevertheless, the study provides information for treating patients who are suspected to be COVID-19 positive and negative as well as those with 
common valvular heart conditions. It discusses the debate and uncertainty of the role of coronary artery bypass graft surgery during the pandemic. Some recommendations, such as the importance of multidisciplinary team discussion, are surely here to stay. Others, for example recommending a $\mathrm{CT}$ of the chest in asymptomatic patients who test COVID-19 negative, are probably impractical or even contraindicated. In the rest, such as whether personal protective equipment affects operating room performance, data will emerge to reinforce or modify them.

At this time of global crisis, national and international associations of cardiac surgeons are co-operating, learning, leading, and sharing information on an unparalleled scale. In many ways we are "feeling our way in the dark." Although useful guidance is provided by the study of Benedetto and colleagues, ${ }^{1}$ the greatest reassurance is the confidence shown by so many senior surgeons that normal cardiac surgery practice will return when the pandemic is over.

\section{Reference}

1. Benedetto U, Goodwin A, Kendall S, Uppal R, Akowuah E. A nationwide survey of UK cardiac surgeons' view on clinical decision making during the COVID-19 pandemic. J Thorac Cardiovasc Surg. 2020;160:968-73.
See Article page 968.

\section{Commentary: Performing cardiac surgery in the coronavirus disease 2019 (COVID-19) era: What is the new normal?}

\author{
Siamak Mohammadi, MD, FRCSC, and \\ Dimitri Kalavrouziotis, MD, FRCSC
}

The global impact of severe acute respiratory syndrome coronavirus 2 (SARS-CoV-2) infection and the coronavirus disease 2019 (COVID-19) pandemic crisis has significantly changed the landscape of cardiac surgical practice. There is a sense of urgency that is all the more intensified given the many unanswered questions surrounding virus transmission. Furthermore, the cardiac surgeon's primary responsibility is to assure patient safety and excellent outcome. There remains a huge gap between the different aspects of this biopsychosocial viral crisis and our current knowledge.

\footnotetext{
From the Department of Cardiac Surgery, Quebec Heart and Lung Institute, Quebec City, Canada.

Disclosures: The authors reported no conflicts of interest.

The Journal policy requires editors and reviewers to disclose conflicts of interest and to decline handling or reviewing manuscripts for which they may have a conflict of interest. The editors and reviewers of this article have no conflicts of interest.

Received for publication May 29, 2020; revisions received May 29, 2020; accepted for publication June 1, 2020; available ahead of print June 11, 2020.

Address for reprints: Siamak Mohammadi, MD, FRCSC, Department of Cardiac Surgery, Quebec Heart and Lung Institute, 2725 chemin Sainte-Foy, Quebec City, Quebec, Canada G1V 4G5 (E-mail: siamak.mohammadi@fmed.ulaval.ca).

J Thorac Cardiovasc Surg 2020;160:977-9

$0022-5223 / \$ 36.00$

Copyright (C) 2020 by The American Association for Thoracic Surgery

https://doi.org/10.1016/j.jtcvs.2020.06.002
}

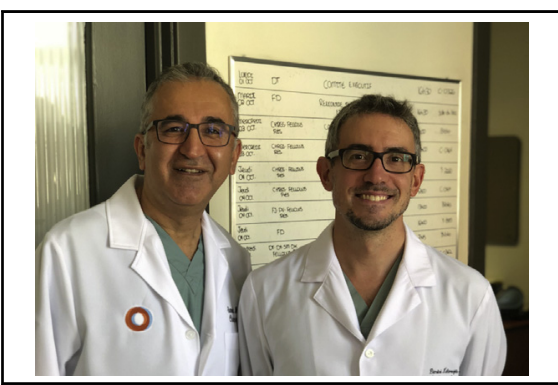

Siamak Mohammadi, MD, FRCSC, and Dimitri Kalavrouziotis, MD, FRCSC

CENTRAL MESSAGE

The optimal preoperative assessment and intraoperative approach to the cardiac surgical patient in the COVID-19 era remain unknown, although information from surveys may be

In this issue useful. of the Journal, Benedetto and colleagues ${ }^{1}$ analyzed the data gathered from a national survey of senior cardiac surgeons across the United Kingdom regarding what needs to be changed in the perioperative approach to the adult cardiac surgery patient during the COVID-19 pandemic. The authors emphasized that expert opinion may help health authorities achieve some interim recommendations while awaiting more robust data. A total of 86 of 198 senior cardiac surgeons of the Society for Cardiothoracic Surgery in Great Britain and Ireland from 35 institutions completed a Web-based questionnaire of 